\title{
Cushing Syndrome
}

National Cancer Institute

\section{Source}

National Cancer Institute. Cushing Syndrome. NCI Thesaurus. Code C2969.

A syndrome caused by high levels of cortisol in the blood either due to excessive

production and secretion of corticosteroids secondary to pituitary or adrenocortical neoplasms, or intake of glucocorticoid drugs. Signs and symptoms include a round face, upper body obesity, fragile and thin skin, purple stretch marks in the skin, fatigue, muscle weakness, hypertension, diabetes mellitus, hypertrichosis and amenorrhea in women, impotence in men, and osteoporosis. 